\section{Integrierter Psychosomatischer Konsiliardienst in der Dermatologie}

Zusammenfassung. Einleitung: 1999 wurde im Zentrum für Hautkrankheiten in Stuttgart-Bad Cannstatt in Kooperation mit einer niedergelassenen Dermatologin und Fachärztin für Psychotherapeutische Medizin ein Psychosomatischer Konsiliardienst eingerichtet. Damit konnte das Behandlungsangebot für die stationären Patienten im Sinne einer psychosomatischen Differenzialdiagnostik erweitert werden und Hilfe zur Krankheitsbewältigung bei somatopsychischen Folgeproblemen chronisch hautkranker Patienten gegeben werden. Methoden: Ziel war die Sensibilisierung der Patienten für ein biopsychosoziales Modell, die Vermittlung von Kratzkontrollstrategien bei Kratzverhalten, die Aufklärung über verhaltensmedizinische Techniken und/oder Konfliktlösungsmodelle sowie konkrete Vermittlung von Therapieplätzen und die Complianceförderung für die somatische Behandlung und psychiatrisch-medikamentöse Empfehlungen. Das Konsiliarangebot wurde ergänzt durch eine theoretische Weiterbildung über allgemeine und spezielle Psychosomatik in der Dermatologie, Gesprächsführungstechniken und eine fortlaufende Balintgruppe für die indikationsstellenden Ärzte. Ergebnisse: In einem Zeitraum von einem Jahr wurden von den behandelnden Stationsärzten 52 Patienten über insgesamt 61 Kontakttermine vorgestellt. 26-mal wurde eine Empfehlung zur stationären oder ambulanten Psychotherapie gegeben, sieben Patienten realisierten sicher den Therapievorschlag. Die Bedeutung des Konsilgespräches für die zugewiesenen Patienten wurde durch die Ärzte als sehr wichtig bis wichtig eingestuft. Fazit: Psychosomatische Differenzialdiagnostik im Rahmen eines integrierten Konsiliardienstes in der stationären Dermatologie ergänzt und erweitert das Behandlungsangebot. Auch in kurzen und einzelnen Kontakten ist es möglich, über eine Vielzahl von Interventionstechniken und das Gespräch über psychotherapeutische Möglichkeiten Krankheitsverständnis und Krankheitsbewältigung günstig zu beeinflussen.

Integrated Psychosomatic Consultation Service in Dermatology. Introduction: In 1999 the Zentrum für Hautkrankheiten in Stuttgart-Bad Cannstatt established a psychosomatic consultation service in co-operation with a local dermatologist who is also a specialist in psychosomatic medicine. This service extended the offering of in-patient treatment by including psychoso-

Akt Dermatol 2001; 27: 187-193

(c) Georg Thieme Verlag Stuttgart · New York ISSN 0340-2541

\author{
Christa-Maria Höring ${ }^{1}$, Gabriele C. Nist ${ }^{2}$ \\ ${ }^{1}$ Hautpraxis, Stuttgart \\ 2 Klinik für Dermatologie und Allergologie im Zentrum für \\ Hautkrankheiten, Krankenhaus Bad Cannstatt \\ (Direktor: Prof. Dr. Weidner)
}

matic differential diagnostic and thus helped chronically ill patients with skin diseases suffering from somatopsychic after-effects to better cope with their disease. Methods: Goals were to sensitise patients for a biopsycho-social model, to offer control strategies for scratch behaviour, to educate on behaviour therapy-techniques and/or on conflict solution models as well as to arrange for therapy, to enhance compliance for somatic treatment and for psychiatric drug treatment. The consultation offering was supplemented by further education on general and specific psychosomatic symptoms in dermatology, interview techniques and by continuous balint group sessions for the ward physicians. Results: Within one year, the resident physicians introduced 52 patients in more than 61 visits. Recommendation for in- or out-patient psychotherapy was given in 26 cases of which seven patients followed through with the recommended therapy. For the ward physicians the importance of the consultation meetings for the assigned patients was estimated very high to high. Conclusions: Psychosomatic differential diagnostic in the course of an integrated consultation service in dermatologic clinics supplies and extends the treatment offering. Even in short and solitary contacts it is possible to improve disease-related insight and coping behaviour by means of various intervention techniques and the information about psychotherapeutic possibilities.

\section{Einleitung}

Nach Untersuchungsergebnissen von Schepank leiden 26\% der Bevölkerung an einer behandlungsbedürftigen psychogenen Erkrankung [1]. Übertragen auf den ambulanten und stationären dermatologischen Sektor können wir hypothetisch von ähnlichen Zahlen ausgehen, wobei sich innerhalb des Fachgebietes Unterschiede zeigen werden. So sind chronische juckende Dermatosen sicher unter psychneuroimmunologischen Faktoren stärkeren Beeinflussungen unterworfen, die sich unter einem Stichwort „Erregung“ zusammenfassen ließen und sind auch eher von Problemen in der Krankheitsbewältigung gefolgt als andere Krankheitsbilder. Es stellt sich die Frage, welche Möglichkeiten es gibt, diesen Anforderungen gerecht zu werden. 
Vor allem in Universitätskliniken haben sich im hautärztlichen Bereich in den letzten Jahren verschiedenste Formen psychosomatischer Konsiliar- oder Liaisondienste etabliert.

Bei einem reinen Konsiliardienst wird der Patient mit einer bestimmten Fragestellung an den Konsiliar weitergeleitet, der wiederum einen Bericht in Form eines zusammenfassenden Arztbriefes über die erhobenen Untersuchungsbefunde erstellt. Ein solcher Konsiliardienst kann seiner Aufgabe nur begrenzt gerecht werden, da die bio-psycho-sozialen Zusammenhänge meist sehr komplex sind. Ein psychosomatischer Liaisondienst hingegen sieht die direkte Kooperation vom Somatiker bzw. dem Behandlungsteam und dem Psychosomatiker auf Station vor. Arbeitsinhalte sind die unmittelbaren Patientenkontakte, Gespräche mit Pflegekräften, Ärzten und Angehörigen, Dokumentation und Arztbriefe, Teambesprechungen, Supervision und die Weiterbildung [2].

Ludwig-Becker [3] berichtet über ihre Erfahrungen nach zweieinhalb Jahren Liaisondienst am Klinikum Aachen. Sie betont die Konstanz des psychosomatischen Ansprechpartners und die Koevolution dermatologisch ärztlichen Denkens mit biopsychosozialem Denken. Während zu Beginn des Dienstes, der zunächst als Konsiliardienst eingerichtet wurde, 16 Konsile pro Jahr angefordert wurden, steigerte sich die Zahl auf 73 vier Jahre später im Rahmen des Liaisonangebotes. Ludwig-Becker führt dies insbesondere auf die informellen interdisziplinären Fallkonferenzen zurück.

Aus dem Zentrum für Dermatologie Gießen berichtet Niemeier [4] über ein Liaisonmodell im ambulanten Bereich, in dem innerhalb von acht Monaten 1,15\% der poliklinisch behandelten Patienten gesehen wurden. Das Modell wurde als Spezialsprechstunde zur Diagnosestellung, Abklärung von Therapiemotivation und Darstellung von therapeutischen Konzepten gestaltet. In insgesamt 87,7\% der Fälle wurde eine Psychotherapie empfohlen. Auch Niemeier diskutiert den Vorteil der engen somatischen und psychosomatischen Kooperation und die Erniedrigung der Schwellenangst, wenn ein psychosomatisches Angebot aus und innerhalb der Dermatologie an den Patienten geht.

Schubert und Bahmer erfragten 1989 [5] die Situation der psychosozialen Versorgung in der Dermatologie an 42 dermatologischen Kliniken. Neben einer großen Variabilität in der Einschätzung psychischer Faktoren hinsichtlich der Ätiopathogenese dermatologischer Erkrankungen wurde deutlich, dass in 23 von 29 in die Auswertung eingegangenen Kliniken psychologische Faktoren berücksichtigt wurden und dass in 34,5\% auch Forschungsprojekte geplant oder in Arbeit waren.

In einer breit angelegten Umfrage von Gieler [6] an dermatologischen Abteilungen in Deutschland stimmten die befragten Mitarbeiter in 55 bzw. 58 von 69 Abteilungen psychosomatischen bzw. somatopsychischen Aspekten im Krankheitsverlauf zu. Hervorzuheben ist die Art der Kooperationsformen: In $71 \%$ wurden Konsile über die Psychiatrie angefordert, in 21,7\% durch festangestellte Psychologen, in 18,8\% im Rahmen eines Liaisondienstes mit Psychosomatik und in 43,5\% wurden Patienten an niedergelassene Psychologen/Psychotherapeuten überwiesen.
Als Aufgabengebiete der Psychosomatik in der Dermatologie wurden die Verbesserung der Krankheitsbewältigung, Diagnostik begleitend zur somatischen Diagnostik, Vorschläge zur Psychopharmakatherapie, Complianceförderung, Aufklärung, Schulungsprogramme und Vermittlung von Entspannungsverfahren hervorgehoben.

\section{Ausgangssituation und Projektplanung}

Das Zentrum für Hautkrankheiten im Krankenhaus Bad Cannstatt umfasst zwei dermatologische Kliniken mit insgesamt ca. 100 stationären und 21 tagesklinischen Betten. Die durchschnittliche Liegedauer der Patienten beträgt etwa acht bis zwölf Tage. Ein Projekt „Psychosomatik in der Dermatologie“ bot sich hier besonders an, da eine Fachärztin für Psychotherapeutische Medizin zur Verfügung stand, welche als beruflich langjährig erfahrene Hautfachärztin mit den dermatologischen Krankheitsbildern besonders vertraut war.

Kliniksleitung, Verwaltung und Konsiliaria entschieden sich für einen zunächst zweiwöchentlich an einem Vormittag auf aktuelle Anfrage stattfindenden Konsiliardienst in den Räumen der Ambulanz ausschließlich für stationäre Patienten in einer Probephase von einem halben Jahr. Da deutlich wurde, dass der strenge 14-tägige Rhythmus der Realität nicht gerecht werden konnte und andererseits eine Akutversorgung organisatorisch nicht notwendig war, einigte sich das Team auf eine flexiblere Lösung mit der Möglichkeit wöchentlicher Vorstellungen. Die Indikation zum Konsiliargespräch wurde durch die jeweiligen Stations-, Ober- und Chefärzte gestellt. Die Patienten wurden ohne Vorgaben ausgewählt. Zeitlich wurden jeweils 30 min für einen Erstkontakt eingeplant, bei Wiedervorstellung auch $60 \mathrm{~min}$. Die zusammenfassende Beurteilung sollte am gleichen Tag ausführlich schriftlich erfolgen; soweit planbar und im Routinebetrieb der Klinik durchführbar, wurden nach den Konsiliargesprächen kurze Gespräche mit den behandelnden Ärzten geführt.

Als inhaltliche Ziele wurden definiert: Herstellen einer therapeutischen Beziehung mit dem Patienten mit Vermittlung eines biopsychosozialen Modells und Einordnen der Relevanz psychosozialer Faktoren für den Krankheitsverlauf und die Krankheitsbewältigung; Formulierung eines möglichen Konfliktes, Verdeutlichung verhaltensmedizinischer Sichtweisen, empathische Förderung eigener Ressourcen in der Krankheitsbewältigung, Vermittlung von ersten Kratzkontrolltechniken und weitere verhaltensmedizinische Strategien im Umgang mit Juckreiz. Es sollte informiert werden über Hilfsangebote wie Entspannung, Schulungsprogramme etc. Wenn nötig, sollte ein wohnortnaher ambulanter Therapieplatz vermittelt werden und ggf. eine stationäre psychosomatisch-dermatologische Rehabilitationsmaßnahme eingeleitet werden. Die psychosomatische Sichtweise sollte in sehr ausführlichen Arztbriefen an die Kollegen eine möglichst breite Darstellung erfahren, um das Verständnis zu fördern und im Denken der Teammitglieder eine Sensibilisierung für psychosomatisch-somatopsychische Fragestellungen anzuregen.

Nach einem halben Jahr wurde das Bedürfnis nach einer Erweiterung des Konsiliarangebotes offenkundig. Da ohnehin im Rahmen der dermatologischen Facharztweiterbildung in 40 Stunden psychosomatische Grundkenntnisse zu vermitteln sind, wurde klinikintern durch die Konsiliaria allen Weiterbil- 
dungsassistenten ein Curriculum angeboten, das spezifisch zum Fach über acht Stunden theoretische Grundlagen der Psychosomatischen Dermatologie, weitere 12 Stunden ärztliche Gesprächsführung und 20 Stunden Balintgruppe beinhaltete. Dabei wurden die ersten beiden Teile in Blockform, die Balintgruppe kontinuierlich und arbeitsbegleitend in zweiwöchigen Abständen über jeweils 2 Doppelstunden angeboten. Dieses Vorgehen hatte den großen Vorteil, dass die erarbeitete Theorie und die Techniken der Gesprächsführung von den Kollegen im Umgang mit und in der Auswahl der Patienten intensiv genutzt werden konnten und anschließend im Rahmen der Balint-Arbeit vertieft und verfeinert wurden. Theorieinhalte bezogen sich auf die psychosomatische Anamnese, den Erstkontakt mit dem Patienten, die Schaffung eines Arbeitsbündnisses zwischen Arzt und Patient, die Diagnose und Differentialdiagnose von psychosomatischen und psychiatrischen Störungen (im Speziellen in der Dermatologie), somatoforme Hauterkrankungen und Krankheitsbewältigungsthemen, psychosomatische Behandlungskonzepte, Kenntnisse verschiedener psychotherapeutischer Verfahren, das Basiswissen allgemeiner Krankheitslehre zu Themen wie Depression und Angst und die Erläuterung des Begriffs der Borderline-Persönlichkeitsstörung.

Die Einübung verbaler Interventionstechniken sollte den Empathiebegriff vermitteln. Die Exploration der subjektiven Krankheitstheorie des Patienten und seiner Behandlungserwartung, das Erlernen von Strategien zur Compliancesicherung, die Klärung von Therapiezielen, der Umgang mit dem schwierigen Patienten, die Erfassung sozialer Überforderungssituationen, Krisenintervention und der Umgang mit Suizidalität waren Schwerpunkte der Ausbildung, wobei insbesondere Signalisierung und Realisierung von Gesprächsbereitschaft trainiert wurden sowie die Rezeption kommunikativer Absichten und emotionaler Gegebenheiten, die Verbalisierung emotionaler Erlebnisinhalte und die Aspekte von Gegenübertragungsphänomenen.

\section{Auswertung und Ergebnisse}

In den ersten sechs Monaten wurden insgesamt 21 Patientenkontakte angefordert. Dabei wurde ein Patient dreimal, drei weitere Patientinnen zweimal vorgestellt, d.h. in 16 Fällen wurde von Seiten der behandelnden Somatiker die Indikation zur ergänzenden psychosomatischen Differenzialdiagnostik und Beratung gestellt.

In den darauf folgenden sechs Monaten wurde der Konsiliardienst 40-mal für 36 Patienten in Anspruch genommen und damit verdoppelt. Anfragen erfolgten von allen Stationen einschließlich der Tagesklinik durch insgesamt zehn Kollegen.

Es ergab sich somit eine Gesamtzahl an Patienten von $\mathrm{n}=52$, davon 39 Frauen zwischen 19 und 81 Jahren (im Mittel 50,8 Jahre), 12 Männer zwischen 28 und 84 Jahren (im Mittel 50,4 Jahre) und ein achtjähriges Kind.

Bei den dermatologischen Diagnosen fanden sich weit an erster Stelle die Neurodermitis und die Psoriasis gefolgt vom dyshidrotischen Handekzem, Pruritus sine materia sowie Urtikaria bzw. Angioödemen (Tab.1).
Tab.1 Dermatologische Diagnosen der vorgestellten Patienten $(n=52)$

\begin{tabular}{lll}
\hline & $\mathrm{n}$ & $\%$ (gerundet) \\
\hline atopisches Ekzem & 9 & 17,3 \\
Psoriasis vulgaris & 7 & 13,5 \\
dyshidrotisches Palmo(plantar)ekzem & 5 & 9,6 \\
Prurigo simplex/Pruriginöses Ekzem & 4 & 7,7 \\
Urtikaria/Angioödem & 4 & 7,7 \\
Pruritus sine materia & 3 & 5,8 \\
Lichen ruber & 3 & 5,8 \\
Lupus erythematodes & 2 & 3,9 \\
neurotische Exkoriationen & 2 & 3,9 \\
hyperkeratotisch-rhagadiformes Handekzem & 2 & 3,9 \\
progressive systemische Sklerodermie & 2 & 3,8 \\
mikrobielles Ekzem & 1 & 1,9 \\
Acne inversa & 1 & 1,9 \\
Shulman-Syndrom & 1 & 1,9 \\
Fibromyalgie-Syndrom & 1 & 1,9 \\
malignes Melanom & 1 & 1,9 \\
Artefakt & 1 & 1,9 \\
Dermatozoenwahn & 1 & 1,9 \\
Leser-Trélat-Syndrom & 1 & 1,9 \\
Lymphödem & 1 & 1,9 \\
\hline \hline
\end{tabular}

Tab. 2 Fragestellungen der hautärztlichen Kollegen auf dem Konsilschein bei $n=52$ Patienten, Mehrfachnennungen möglich

\begin{tabular}{lr}
\hline Mitbeurteilung erbeten/Procedere & $15 \times$ \\
Hinweise auf Depression oder Angst & $10 \times$ \\
Krankheitsbewältigungsproblematik & $15 \times$ \\
auffällige psychosoziale Belastung & $13 \times$ \\
Suchtproblematik & $4 \times$ \\
Kratzkontrolle und Juckreizbewältigung & $5 \times$ \\
\hline
\end{tabular}

In den Konsiliaranforderungen wurde in 15 Fällen explizit nach einer Mitbeurteilung und dem weiteren Procedere bei auffälligem Leidensdruck gefragt (Tab.2). Ebenfalls 15-mal wurde Hilfe bei der Krankheitsbewältigung erbeten, 4-mal aus Gründen mangelhafter Compliance und zur Suchtberatung, 13-mal wurden stichwortartig auffällige psychosoziale Belastungsfaktoren beschrieben, 10-mal wurde auf erkennbare Depressivität und Angst verwiesen. Kratzkontrollstrategien als Unterstützung zur laufenden Behandlung wurden 5-mal erfragt (Tab. 2).

Die Mitbeurteilung galt im Wesentlichen der differenzialdiagnostischen Einordnung und Überlegungen zur differenziellen Therapieindikation, Konflikterarbeitung bzw. Fokusformulierung (Tab. 3 u. 4). Die in Tab. 3 genannten Interventionen spiegeln das Spektrum und die Möglichkeiten (psycho-) therapeutischer Maßnahmen im Rahmen des Konsiliardienstes bei ein bis maximal drei Kontakten pro Patient wider. 
Tab. 3 Therapeutische Interventionen, unabhängig von der dermatologischen Diagnose

- Sensibilisierung für biopsychosoziale Zusammenhänge, Aufstellen einer Baseline (z. B. „Sie haben eine Atopie. Sie können ein Ekzem bekommen." „Was bedeutet die Erkrankung für Ihr tägliches Leben?")

- Information über Forschungsergebnisse der Psychosomatik in der Dermatologie

- Erarbeiten eines deutlichen Konfliktes/Fokusformulierung

- Benennung von Krankheitsbewältigungsstrategien, Flexibilisierung der Copingstrategien

- kognitive Neubewertung, Aufmerksamkeitsumlenkung z. B. bei Dysästhesien, Schmerz, Juckreiz

- Vermittlung von Kratzkontrollstrategien

- Complianceförderung für aufwendige oder neue Therapien

- Krisenintervention bei ausgeprägter depressiver Reaktion oder Angst

- Vermittlung von Therapieplätzen

- Aufklärung über Therapiemodelle, Entwicklung einer (psycho)therapeutischen Perspektive

- medikamentöse Empfehlung, Entängstigung bezüglich der Einnahme von Psychopharmaka

Tab. 4 Beispielhafte, auf die dermatologische Diagnose bezogene (psycho)therapeutische Interventionen

juckende Dermatosen, die mit Kratzverhalten einhergehen

Urtikaria/Angioödeme

Palmoplantarekzeme, Psoriasis

Autoimmunerkrankungen
Techniken der Verhaltensmedizin, Kratzkontrolle, kognitive Strategien, Selbstmanagement, Complianceförderung, Konflikterarbeitung, Kommunikationstraining, Hinweise auf Schulungsinhalte für Neurodermitis etc., auf Stressbewältigungstraining

Konflikterarbeitung, Korrektur irrationaler Überzeugungen wie einseitige Attribution auf eine Psychogenese

\section{Krankheitsbewältigung}

Erarbeiten krankheitsbezogener Attributionen und Kontrollüberzeugungen, Korrektur illusionärer Erwartungen, Vermittlung von Selbsthilfegruppen, Literatur

Patienten mit Hauterkrankungen, Psychotherapieempfehlung deren Symptom zum „Leitsymptom" wird oder die die Zeit in der Hautklinik nutzen, um gravierende psychosoziale Probleme zur Sprache zu bringen

Im Einzelnen wurde 16-mal eine Empfehlung zur ambulanten Psychotherapie gegeben, 6-mal zur stationären Psychotherapie, 4-mal eine Weiterleitung an die psychotherapeutische Ambulanz für Ältere eines hiesigen Ausbildungsinstitutes (Tab.5). In acht Fällen wurden Entspannungsverfahren, 11mal erste Kratzkontrolltechniken vermittelt, 3-mal andere Verhaltenstherapietechniken oder Interventionen zur Complianceförderung.
Tab. 5 (Psycho)Therapeutische Empfehlungen bei $\mathrm{n}=52$ Patienten

$\begin{array}{lr}\text { Empfehlung zur ambulanten Einzel-Psychotherapie } & 16 \times \\ \text { Empfehlung zur primär stationären Psychotherapie } & 6 \times \\ \text { psychotherapeutische Ambulanz für Ältere (Gruppe) } & 4 \times \\ \text { Psychopharmaka } & 11 \times \\ \text { Entspannungsverfahren } & 8 \times\end{array}$

Insgesamt wurde also 26-mal, d.h. in 50\% der Fälle eine Psychotherapieempfehlung im engeren Sinn gegeben. Die Patienten, denen eine Psychotherapieempfehlung gegeben wurde, wurden, soweit sie nicht durch die Untersucherin selbst in die Therapie übernommen wurden, angeschrieben, ob das Angebot realisiert wurde. Eine Patientin hatte bereits im Vorfeld eine Psychotherapie abgelehnt. Vier weitere antworteten auf die Anfrage und begründeten ausführlich die Nichtaufnahme zum Teil mit einer langen Therapieplatzwartezeit. Diese Patienten berichteten zusätzlich, dass das Gespräch ihnen wichtige Anstöße geliefert habe. Vier Patienten nahmen ein stationäres Therapieangebot an, einer davon zusätzlich die ambulante Fortführung, vier weitere Patienten begannen eine ambulante Psychotherapie. Das heißt insgesamt 37,7\% der Patienten, für die eine Therapieindikation gestellt wurde, nahmen das Angebot wahr. 13 Patienten antworteten nicht auf die Anfrage; es ist wohl davon auszugehen, dass diese Patienten die Therapieempfehlung nicht angenommen haben.

Die kontinuierliche Betreuung der Kollegen durch immer die gleiche Psychosomatikerin, das gemeinsame Verständnis dermatologischer Inhalte und die Möglichkeit zur direkten Rücksprache förderten die Arbeit im Sinne eines Liaisondienstes, der hier jedoch als integrierter Konsiliardienst bezeichnet werden soll, da eine auch perspektivisch angelegte institutionalisierte Teamsupervision (noch) nicht zustande kam, wenngleich in größeren Abständen fraktioniert angebotene Weiterbildungstage avisiert sind und die Balintgruppe fortgeführt wird.

Die intensive Weiterbildung schlug sich eindrucksvoll in der Formulierung der Konsiliaranfragen nieder. Anfangs fehlten Konsiliarscheine manchmal ganz oder es wurden spärliche Bemerkungen wie „ungewöhnliche Urtikaria mit Angioödem, psychotherapeutische Mitbeurteilung erbeten“ im Begleitbrief mitgegeben. Später formulierten die meisten Kollegen die Anfragen ausführlich und differenziert und somit für die Konsiliaria sehr hilfreich. Dies hatte damit zu tun, dass in den BalintGruppen viel über die Bedeutung von Übertragungs- und Gegenübertragungsphänomenen und über das Bemühen eines Patienten, in der Interaktion oft seine eigentliche Not indirekt dazustellen sowie über Techniken eine therapeutische Beziehung herzustellen, gesprochen worden war. Ein Beispiel mag dies verdeutlichen: „Patientin, 64 Jahre alt mit hyperkeratotisch-rhagadiformem Palmoplantarekzem. Patientin sehr leidend, auf klare Fragen ausweichende Antwort. Familiäre Belastung (geschieden, 1 Kind Apoplex, 1 Kind Narkolepsie, 2 Umschulungen wegen Mehlallergie). Sieht selbst Zusammenhang zur Psyche. Aggressivität bei mir vorhanden, aber in Grenzen und nicht die ganze Zeit.“

Die Beurteilung der indikationsstellenden Kollegen und Kolleginnen wurde nach $1 \frac{1}{2}$ Jahren mittels eines strukturierten Fra- 
Tab. 6 Fragebogen zur Erfassung der Zufriedenheit mit dem Konsiliardienst für ärztliche Mitarbeiter

\begin{tabular}{|c|c|c|}
\hline Fragen & Mittelwert & SD \\
\hline $\begin{array}{l}\text { 1. Welche Bedeutung schreiben Sie dem psychosomatischen Konsil für die von Ihnen } \\
\text { vorgestellten Patienten zu? }\end{array}$ & 1,67 & 0,52 \\
\hline $\begin{array}{l}\text { 2. Wie wichtig ist Ihnen die Erstellung eines besonders rasch zugestellten } \\
\text { Konsiliarberichtes? }\end{array}$ & 2,17 & 0,75 \\
\hline 3. Wie gut verständlich ist die auf Psychosomatik ausgelegte Sprache der Berichte? & 2,33 & 0,82 \\
\hline 4. Wie beurteilen Sie die inhaltliche Aussagekraft bezogen auf Ihre Patienten? & 2,33 & 0,52 \\
\hline 5. Konnten Sie die Empfehlungen nachvollziehen? & 1,83 & 0,41 \\
\hline 6. Wie groß war die Entlastung durch die Mitbeurteilung für Sie persönlich? & 2,83 & 1,33 \\
\hline $\begin{array}{l}\text { 7. Glauben Sie, dass die Erarbeitung psychosozialer Zusammenhänge und } \\
\text { gegebene Therapieempfehlungen für die Gesamtbeurteilung des Krankheitsverlaufes } \\
\text { wichtig sind? }\end{array}$ & 2,0 & 0,63 \\
\hline 8. Für wie wichtig halten Sie die angebotene Weiterbildung Psychosomatik? & 2,33 & 0,82 \\
\hline 9. Für wie wichtig halten Sie die Balintgruppe? & 2,33 & 0,82 \\
\hline $\begin{array}{l}\text { 10. Für wie wichtig würden Sie aufgrund der bisherigen Erfahrungen eine } \\
\text { Teamsupervision halten? }\end{array}$ & 2,17 & 0,75 \\
\hline
\end{tabular}

Beurteilungskriterien: 1 = sehr/sehr viel; 2 = viel; 3 = mittel; 4 = wenig; 5 = gar nicht/keine

gebogens erfasst (Tab. 6). Dieser Fragebogen wurde nur für die Beurteilung dieses Konsiliardienstes entworfen und ist deshalb nicht mit anderen Fragebogen vergleichbar. Zwei von acht abgegebenen Fragebogen waren nicht auswertbar, da die Einordnungen unvollständig waren oder ohne genaue Bezifferung kommentiert wurden.

Die Bedeutung des psychosomatischen Konsiliardienstes für die Patienten wird mit einem Mittelwert von 1,67 insgesamt als sehr hoch eingeschätzt und war bei einem Mittelwert von 1,83 für die Behandler auch gut nachvollziehbar. Auffällig ist, dass die persönliche Entlastung mit einer Mittelwertangabe von 2,8 von den Kollegen als relativ niedrig, zum mittleren Bereich tendierend beurteilt wird. Dies könnte damit zusammenhängen, dass mit dem Einführen des Konsiliardienstes zunächst eine höhere Arbeitsbelastung auf die Kollegen zukam, da Fortbildung und Balint-Arbeit viel Zeit kosteten und es unter Umständen auch anstrengend war, die von der Konsiliaria vorgeschlagenen Interventionen umzusetzen oder fortzuführen. Hier wäre es hilfreich, nach einem längeren Zeitraum eine neue Evaluation vorzunehmen, wenn die Arbeitsweise im Gesamtkonzept der Klinik noch selbstverständlicher geworden ist.

Die Bereitschaft der Kollegen (Item1), sich auf psychosomatische Fragestellungen einzulassen, ist sehr hoch, zumal die psychosomatische Sichtweise sehr gut bis gut nachvollzogen werden konnte und die Empfehlungen in das Gesamtbehandlungskonzept einbezogen wurden. Auch die Items 3 und 4, die sich auf die Verständlichkeit und Aussagekraft beziehen, müssten nach einer längeren Dauer des Konsildienstes noch einmal aufgegriffen und mit dem vorhandenen Ausbildungsstand der Kollegen in Psychosomatik in Zusammenhang gestellt werden. Die geringe Abweichung in der Beantwortung des Items 10 im Vergleich zu den Items 8 und 9 wurde dahingehend interpretiert - und dies war auch in den laufenden Balint-Gruppen erfahrbar - dass die Kollegen einen Bedarf haben für konkrete Handlungsanweisungen und die Einübung spezieller Interventionstechniken („wie mache ich es genau“).

\section{Diskussion}

Die Integration der Psychosomatik im Fach Dermatologie ist inzwischen nach vielen Jahren „Pioniersarbeit“ über die Etablierung psychosomatisch-dermatologischer Rehabilitationskliniken, die zunehmende Einbindung in den universitären Bereich mit Forschungsprojekten, einzelne Modelle an städtischen Hautkliniken, die geforderten Weiterbildungsinhalte in der Facharztausbildung, zentrale und regionale Tagungen und über die Tätigkeit des Arbeitskreises Psychosomatische Dermatologie APD in der Deutschen Dermatologischen Gesellschaft auch institutionell erkennbar.

Psychosomatische Dermatologie, wie sie derzeit in der Leitlinienkonferenz definiert und ausgearbeitet wird, bezieht sich zum einen auf psychosomatische Dermatosen, also Hautkrankheiten, bei denen psychosomatische Aspekte in der Entstehung oder Verarbeitung eine bedeutende Rolle spielen wie Neurodermitis, Psoriasis, Urtikaria, aber auch auf psychiatrische Erkrankungen wie Wahnsyndrome und auf die Haut bezogene Erkrankungen, die als somatoforme Störungen eingeordnet werden. Auch Hauterkrankungen, bei denen somatische Aspekte im Vordergrund stehen, wie Hauttumoren, entstellende Dermatosen usw. werden erfasst. In unserer Arbeit sollte nicht schwerpunktmäßig auf das psychopathologische Diagnosenspektrum eingegangen werden, da dieses vorhandene Publikationen nur unwesentlich ergänzt hätte [4]. Die Diagnosen aus psychosomatischer Sicht reichten auch bei uns von schweren Ich-strukturellen Störungen über psychiatrische Diagnosen sowie neurotische Störungen bis zur Beeinträchtigung der Lebensqualität bei normal neurotischer Struktur.

Psychosomatik in der Dermatologie meint vor allem auch Hautveränderungen als Leit(d)symptom, die klinisch minimal sein können, aber als Präsentiersymptom gravierende psychosoziale Belastungsfaktoren zur Sprache bringen.

Die Bemühungen von psychosomatischen Therapieansätzen in der Dermatologie [4], von integrativer Kurzzeitpsychotherapie bei Hauterkrankungen [8] und von psychosomatisch-dermato- 
logischer Rehablitationsbehandlung [9] zielen dahin, die Psychosomatik bei psychosomatischem Mitbehandlungsbedarf [7] möglichst eng an die Somatik anzubinden und einen diagnostischen und therapeutischen „Dialog“ zu entwickeln, in dem es gilt, den verschiedensten Aspekten gerecht zu werden. Ein Aspekt ist das Wissen um die Häufigkeit psychischer und sozialer Einflüsse auf die Erkrankung: Schaller fand in 21\% seiner 249 untersuchten erwachsenen Patienten einen solchen Einfluss, Niemeier berichtet über $18 \%$ der Hautpatienten einer Routineambulanz, bei denen sich die Diagnose einer somatoformen Störung fand. Für 81 in der Psychosomatischen Sprechstunde vorgestellte Patienten gab er in 82,7\% eine Empfehlung zur Psychotherapie, eine Zahl, die sich am ehesten aus der hohen Professionalität der Sprechstunde erklären lässt und aus einer bereits zuvor stattgefundenen Selektion innerhalb des gut ausgebildeten Gießener Teams.

Ein psychosomatisches Konsil sollte insbesondere dann angefordert werden, wenn der behandelnde Arzt eine auffällige Diskrepanz zwischen objektivem und beklagtem Befund wahrnimmt, wenn eine schwer nachvollziehbare Therapieresistenz zu beobachten ist und/oder Complianceprobleme auftreten, wenn offen oder angedeutet psychosoziale Belastungsfaktoren benannt und aus der Anamnese nachvollzogen werden oder wenn der Patient als „schwieriger Patient“ erlebt wird und in der Interaktion durch den Behandler bei sich selbst eher heftige emotionale Reaktionen erlebt werden.

Ein ausgearbeitetes psychotherapeutisches Konzept im Rahmen einer dermatologischen Abteilung sollte ein vom Team getragenes breites Spektrum an Angeboten beinhalten wie die Vermittlung von Entspannungstechniken, Familiengespräche, Schulungsinhalte, um nur einige Möglichkeiten zu nennen, die auch innerhalb einer relativ kurzen Liegedauer realistisch sind. Die einzelnen psychotherapeutischen Interventionen, wie sie in einer dermatologischen Abteilung geleistet werden können, sind genauso wegweisend wirksam wie das Erlernen einer adäquaten Lokaltherapie, die richtige Verbandstechnik und das Verständnis für allergologische Zusammenhänge im Rahmen der Aufklärung.

Der Weg selbst muss im ambulanten Bereich weiterverfolgt werden. Der große Vorteil eines wohnortnahen Konzeptes liegt darin, dass für Patienten, denen eine Psychotherapieempfehlung gegeben wird, Therapieplätze vermittelt werden können.

Bezogen auf das Stuttgarter Projekt zeigte sich, dass die Möglichkeit der konkreten Übernahme in Therapie durch die Konsiliarärztin selbst die geringste Hemmschwelle darstellt, da bereits eine therapeutische Beziehung „gezündet“ wurde. Für eine psychotherapeutisch tätige Dermatologin mit besonderem Interesse an diesem Fach entwickelt sich eine zweifache Chance, mit dem Kranken ein Bündnis einzugehen: die individuelle persönliche Beziehungsgestaltung und die besondere fachliche Motivation, die von Patienten gewürdigt wird, da sie sich in ihrem Anliegen, sich somatisch und seelisch verstanden wissen zu wollen, rascher wiederfinden. Aufklärungsarbeit über Zusammenhänge zwischen Angst und Allergie [10], über verhaltensmedizinische Schulungsprogramme [11] und über Zusammenhänge zwischen der Art des Kommunikationsverhaltens und der Intensität und Rückfallhäufigkeit eines Neurodermitisschubes [12] wird geleistet.
Schepank zieht in seiner Arbeit über den Versorgungsbedarf psychogen Kranker in der Bevölkerung u.a. folgendes Fazit [1]: Es leite sich ein Aufgabenkatalog ab, der die nächsten Dekaden beschäftigen werde: Die psychosomatische Ausbildung an der Universität und in der Facharztweiterbildung, eine verstärkte Einrichtung von Konsil- und Liaisondiensten, die Koordination mit der ambulanten Versorgung, Forschung, primärpräventive Gesichtspunkte und Öffentlichkeitsarbeit.

Mit dem Stuttgarter Projekt wird dazu einen Beitrag geleistet, der von allen Beteiligten geschätzt wird. Im Unterschied zu Welzel-Ruhrmann [13], die aus dem ersten Jahr des beobachteten Konsiliarzeitraumes eine Zuweisung von Patienten speziell über eine Station beschreibt, wurde hier von Beginn an das Angebot von allen Stationen angenommen, so dass von einer guten Integration in die Gesamtklinik ausgegangen werden muss, die nicht personenabhängig ist. Dies liegt sicherlich nicht zuletzt an der personellen Konstanz im Beobachtungszeitraum.

Die Bestrebungen gehen dahin, die bestehende Arbeit als festen Bestandteil zu konsolidieren und sich Gedanken zu machen über Weiterentwicklung und Ausbau des Modells, über Ergänzung und Einbeziehung des nichtärztlichen Teams.

Günstig für eine solche Konsolidierung und Etablierung ist in jedem Fall die Doppelqualifikation der Konsiliaria, die personelle Konstanz, die räumliche Institutionalisierung in der Hautklinik selbst, die flankierenden Weiterbildungsmaßnahmen und die Balint-Arbeit. Personelle Ressourcen innerhalb der Klinik wären günstig zur Etablierung eines Entspannungsangebotes oder Schulungsprogramms. Dies erscheint in Stuttgart aussichtsreich, da zur Hautklinik eine Tagesklinik gehört, in die ein solches Konzept integriert werden könnte.

Wenn sich an der Haut im Sinne eines Haut-Ichs nach D. Anzieu die vielfältigen Ich-Funktionen des Menschen wiederfinden lassen, dann kann es uns als Dermatologen mit psychosomatischem Anliegen nur locken, dies auch in unser Behandlungskonzept einzubeziehen. Der stationäre Rahmen bietet dazu die nötige Zeit. Diese Chance gilt es zu nutzen.

\section{Literatur}

${ }^{1}$ Schepank H. Die Versorgung psychogen Kranker aus epidemiologischer Sicht. Psychotherapeut 1994; 39: 220-229

2 Pontzen W. Psychosomatischer Konsiliar- und Liaisondienst. Psychotherapeut 1994; 39: 322 - 326

${ }^{3}$ Ludwig-Becker F, Altmeyer S, Petzold ER. Evaluation des psychosomatischen Liaisondienstes in der Dermatologie des Universitätsklinikums Aachen. Unveröffentlichte Mitteilung (Information zu beziehen über die Autorin direkt)

${ }^{4}$ Niemeier V, Kupfer J, Köhnlein B, Schill WB, Gieler U. Der psychosomatische Therapieansatz in der Dermatologie. H+G 1996; 71 : 902-907

${ }^{5}$ Schubert HJ, Bahmer F. Stellenwert und Berücksichtigung klinisch-psychologischer Erkenntnisse in der Dermatologie. Akt Dermatol 1989; 15: 69-72

${ }^{6}$ Gieler U, Niemeier V, Kupfer J, Brosig B, Schill WB. Psychosomatische Dermatologie in Deutschland - eine Umfrage an 69 Hautkliniken. Hautarzt 2001; 52: 104-110

${ }^{7}$ Schaller C, Alberti F, Pott G, Ruzicka T, Tress W. Psychosomatische Störungen in der Dermatologie - Häufigkeiten und psychosomatischer Mitbehandlungsbedarf. Hautarzt 1998; 49: 276-279 
${ }^{8}$ Simmich T, Traenckner I, Gieler U. Integrative Kurzzeitpsychotherapie bei Hauterkrankungen. Hautarzt 1998; 49: 203 -208

${ }^{9}$ Wehrmann J. Indikationen für eine psychosomatisch-dermatologische Rehabilitationsbehandlung. Hautarzt 1996; 47: 253-257

10 Schmid Traub W. Vermehrt Angst und psychische Auffälligkeiten bei Allergikern. Allergologie 1995; 18: $13-19$

${ }^{11}$ Gieler U, Bräuer J, Freiling G. Neurodermitisschulung - ein neuer psychosomatisch orientierter Behandlungsansatz. In: Gieler U, Stangier U, Brähler E (Hrsg). Hauterkrankungen aus psychologischer Sicht. Jahrbuch der Mediz Psychol, 1993

12 Ehlers A, Osen A, Wenninger K, Gieler U. Atopic dermatitis and stress: possible role of negative communication with significant others. Int J of Behav Med 1994; 1: 107-121

${ }^{13}$ Welzel-Ruhrmann C, Wermter F, Liedtke B. Katamnestische Evaluation eines psychosomatisch dermatologischen Konsiliardienstes. Psychotherapeut 1999; 44: 220-226

\section{Dr. med. Christa-Maria Höring}

Hautärztin, Allergologin und Fachärztin für psychotherapeutische Medizin

Schlossstraße 100

70176 Stuttgart 